\title{
Ebola outbreak claims more than 60 lives
}

\author{
Anne Gulland
}

London

Doctors are fighting to contain an outbreak of Ebola haemorrhagic fever in Guinea that has claimed more than 60 lives and is now being reported in the capital, 400 miles away. As of 27 March, the World Health Organization said that there had been 103 suspected and confirmed cases of the disease, including 66 deaths. WHO said it could not confirm all cases because of the rapidly changing situation.

Most of the cases have been reported in three districts in the south east of the west African country, Guéckédou, Macenta, and Kissidougou, but five cases have also been reported in the capital, Conakry. Neighbouring Liberia and Sierra Leone have also reported cases of the disease among people who had previously travelled to Guinea: 16 cases in total, including 11 deaths.

The outbreak is being controlled by the country's ministry of health, alongside WHO and other international agencies, including the charity Médecins sans Frontières. Armand Sprecher, public health specialist at the charity, said that teams on the ground in Guinea were controlling the disease but the future was uncertain.

"We don't know if the cases in Conakry trace back to Guéckédou. If we can find an epidemiological link between the cases we can get a handle on it. But we don't know if we're going to get multiple points of entry of the disease and how many points of entry there will be," he said.

In conjunction with the ministry of health the charity has set up a 10 bed facility in Guéckédou and a six bed facility in Macenta. It is also about to set up a unit in Conakry. Médecins sans Frontières is also identifying people who have been in contact with Ebola patients and are showing symptoms of the disease.
Ebola is highly contagious and can be spread through contact with bodily fluids such as blood, urine, sweat, and mother's milk. Workers have been given protective suits, gloves, masks, and goggles, and decontamination chambers have been installed between the isolated patients and the external environment.

"In the past healthcare staff have turned and fled but that doesn't seem to be happening this time. They are developing confidence in the protective techniques we use," said Sprecher.

The last known outbreak of the disease was in Uganda and the Democratic Republic of Congo, and killed dozens of people. Sprecher classified the current outbreak as "medium sized."

The disease's mortality rate is up to $90 \%$ and it is thought to be carried by bats, a local delicacy whose sale and consumption has now been banned by the Guinean government. There is no known treatment for the disease and care is supportive in the form of rehydration and pain relief.

"We follow the model for septic shock as there is good microbiological evidence that septic shock and Ebola follow similar paths," said Sprecher.

Scientists from the Institut Pasteur in Lyon, France and Dakar, Senegal and the Bernhard-Nocht Institute of Tropical Medicine in Hamburg, Germany, have been testing samples in Guinea. The disease initially presents as an influenza type illness with fever and then vomiting and diarrhoea. It is not until its advanced stage that heavy bleeding from the nose or in urine occurs.

"Having labs on the ground is really important as it means that we can put people with the disease into the treatment units," said Sprecher.

Cite this as: BMJ 2014;348:g2473

๑ BMJ Publishing Group Ltd 2014 